tionne donc en quelque sorte comme un rhéostat bien gradué.: La pratique. a d'ailleurs montré qu'il en était bien ainsi et qu'il était possible de prévoir, s'il le fallait, l'adoption de soupapes pour lesquelles la résistance combinée alteigne seulement 70 ohms.

Enfin, une des caractéristiques de la soupape Giles esti de faciliter l'amorçage des distances explosives d'une même colonne. Fn effet, le nombre d'intervalles d'air de cet appareil est tel que la surtension d'amorçage scrait considérable s'il n'y avait pas un dispositif spécial prévu à cet effet. Ce dernier consiste à reporter successivement la totalité de la tension du réseau sur chacune des distances explosives, de telle sorte qu'on amorce celles-ci les uncs après les autres au lieu de les amorcer toutes en même temps, comme cela a lieu dans les autres appareils.

Il nous reste à ajouter un mot sur le groupements ou mode de connexion donné habituellement à ces soupapes ; comme les surtensions ì basse et moyenne fréquence peu-

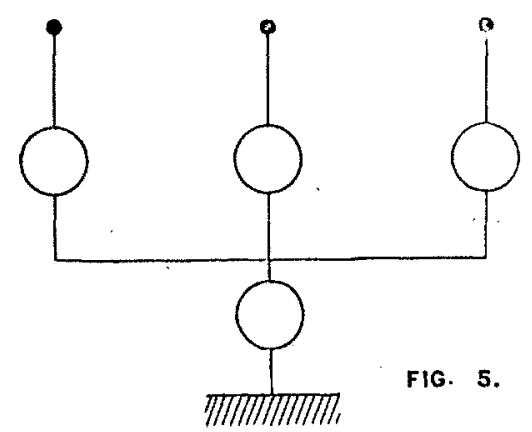
vent sc faire sentir aussi bien entre phases qu'entre chaque phase ret la terre, il sera préférable d'adopter le montage par 4 ide la figure 5. Ainsi qu'on peut le voir, cette disposition a, comme avanlage, d'opposer aux surtensions toujours deux appareils en séric, calculés

pour la moitié de la tension normale, qu'elles se produisent entre conductcurs ou entre chaque conducleur et la terre.

Avant de terminer, je crois devoir dire un mol sur la question de la misc à la terre du point neutre dans les installations à courants triphasés. Cettc question a toujours préoccupé installateurs ct exploitants el a été, depuis longtemps, le sujet de nombreuses controverses.

Des nombreuses expériences qui ont été failes avec l'un ou l'autre système, et que nous avons pu observer personnellement, nous croyons pouvoir dire, sans être contredil par personnc, que la mise directe du neutre à la terre présente trois sérieux inconvénients :

$r^{\circ}$ Augmentation du danger de mort, au cas où quelqu'un vient ì touchcr des conducleurs sous courant;

$x^{\circ}$ Mise en court-circuit directe sur la machine de la phase qui scrail mise accidentellement ì la terre, ce qui enlève toule possibilité évenluelle de marcher avec les deux autres phases, s'il y a un défaut d'isolement à la troisième;

$3^{\circ}$ Perturbations téléphoniques très forles dans les réseaux de ville à câbles souterrains, ces dernières reudant même l'exploitatión téléphonique impossible si les alternateurs ont des harmoniques de denture d'ordre $3\left({ }^{1}\right)$.

Toutefois, comme la mise du neutre à la tere présculc de nombreux avanlages, nous préconisons de la réaliser par. l'intermédiaire d'une résistance hydraulique, ou par un artifice de ce genre, ce qui supprime, par conséquent, la plupart des inconvénients que nous venons de citer. Il ne nous rst malheureusement pas possible de nous élendre plus longuement sur ce point dans une causerie où l'on ne s'occupe que de la question de la protection des réscaux contre les surtensions.

Bruxelles, le 23 mars 1912.

(1) Voir La Revue électrique, t. XI, 30 awril rgog. r. 304.

\section{SERVICE D'ÉTUDES DES FORGES HYIRAULIOUES DANS LA RÉGION DES PYRÉNÉES}

Nous avons tenu nos lecleurs au courant de l'organisation du Service d'études des grandes Forces hydrauliques, ct des résultats obtenus dans la région des Alpes. Inulile d'en faire, une fois de plus, ressortir toule l'importance, car il n'est persomnc qui, ayant à s'occuper de chutes d'eau, n'ail cu recours aux précieux documents publiés par le Service.

On sait qu'il a été étendu aux cours d'eau des Pyrénées, et prochainement nous nous proposons de donner ici une analyse des travaux ellectués dans cette région. Mais, sains attcndre davantage, nous teinons à signaler la publication qui vient d'être faite en librairie des premiers résultats obtenus au 3 I décembre s 9 ro.

Ils occupent deux tomes des Annales de la Dircction de J'Hydraulique agricole. Le premier, de 260 pages, illustré de superbes vues photographiques, contient les rapporls de M. R. Tavernier, inspecteur général et de M. Matterke ingénieur en chef des Ponts el Chaussées, sur les opérations faites, puis les résultats obtenus pour le bassin de l'Adour. Le tome II est formé de 5 ro pages de barêmes et courbes de jaugeages, plus 62 graphiques de débits des cours d'eạ du bassin de la Garonne.

Nous pensons que ce renscignement sera ulile à plus d'un lecteur, car les Pyrénćes, tout d'abord délaissées pour les Alpes, exportant leur houille blanche par de grandes lignes de transport, semblent par contre devoir etre bienlôt le pays d'ćlection du four ćlectrique, apte à faire mettre en valeur leurs richesses minérales très nombreuses.

\section{LA DURANCE ET SON UTILISATION POUR L'AGRIGULTURE ET L'INDUSTRIE}

Nos lecteurs se rappellent certainement les éludes publiéco ici sur celle question par $M$. Wulherm, ingénieur en chel des Ponts et Chaussées, à Grenoble. Dans les numéros dé février, mars, mai et décembre rgı, l'inlassable et savant ingénieur, à qui ses travaux si jmportants sur le régime des lorrents valent une inconlestable autorité en matière d'amínagement des rivières alpestres, nous a donné avec un apergu de l'utilisation actuclle des eaux de la Durance; l'esquisse des grands projets de régularisation dont ce cours d'eau esl susceptible pour le plus grand bien de l'agriculhire en Provence et de l'industric dans la zone de notre graid poit méditerranécn.

" L'ouvre qui a été réalisée, disail oxcellemment M. Wrtarela dans les conclusions résumant celte séric darticles, est considérable, mais elle n'est pas lerminéc. Is irrigations ont pris un lel développement que l'can de lit Durance devient souvent insuffisante, el cependant il y a d? nouveaux besoins qui sc manifestent. L'industric we ta houille, blanche vient à peine de naître et déjà clle réclame une négularisation du régime de la capricieuse rivièrc. L L même question se pose d'aillcurs plus ou moins pour tous les cours d'eau des Alpes, car partout l'industrie des grandes forces hydrauliques souffre du manque d'cau, surtout pertdant l'hiver. C'est done à juste titre que l'on se préoceupe de remédier à cetle facheuse situation. Malheureusement, if scra lc plus souvent impossible d'arriver à une solution 
pratique de ce problème si complexe ; mais c'est une raison de plus pour agir là ou les circonstances sont favorables... " Pour déterminer l'action, M. Whrem a eu l'idée de faire connaître au grand public les termes du problème dont il a éludié la solution, et il l'a fait sous. la forme d'un livre. Ce liyre est avant tout une œuvre d'ingénieur, mais non point ì la manière aride et sèche que lui aurait donné un technicicn sans souci de faire se dégager des documents qu'il produit cette éloquence persuasive qui rallie le lecteur à la cause plaidéc. l.e livre est d'un savant qui se fait modeste et d'un apôtre communiquant sa foi en l'utilité de l'ouviré qu'il préconise. Nous ajouterons qu'il est aussi d'un parfait écrivain par le charme du style qui, en certaines pages, est en complète harmonie avec la beauté des sites.... que l'art de l'iǹgéníur ne profanera point.

J'ouvrage a pour titre : "LA DuRancé ; Elude de l'utilisálion de ses eaux et de l'amélioration de son régime par la création de barrages. "Il débute par uile très complete monographie du bassin de la Durance, puis traite des irrigations et parle des usines, pour aborder ensuite l'objet principal : la régularisation du régime de la Durance. Viennent alors les projets des barrages de Serre-Poncon; du Guil ol de l'Ubaye, l'aménagement du Verdon et la dérivation de la source de Fontaine-l'Evêque.II se terrinine par l'expóséd'uin progiramme d'aménagement rationnel des eaux du bassin de la Durance.

Co sont les idées directrices de cette ceuvire, tracéc par l'éminent collaborateur de celle Revue, qư chin fidèle observaleur du programme de La Houille Blanche, nous nous proposons de faire connaître à ses lecteurs.

Aliparavant, ouvrons úne parenthèse poử leur signaler gue le livre de M. Wirhel.M; qui comporte environ 360 pages de grand format, est splendidement édité ; de très nombreuses phototypies et un grand panorama illustrent le texle complélé par: une carte en couleur ; tous ces éléments rcvếtent diune belle forme artislique ce travail d'ingénieur $\left({ }^{1}\right)$.

\section{RÉGULARISATION DE LA DURANCE}

\section{LA SOLUTION DES GRANDS BARRAGES}

Tous les cours d'eau ont un régime variable suivant les saisons, avec des écarts plus ou moins grands entre les dúbits d'étiage, les débits des eaux moyenncs ét les débits des crues. On connaît les inconvénients qui en résultent pour les usines hydroélectriques, qui sont toujours aménagées pour un débit au moins double de celui de l'étiage, quand ce n'est pas le triple ou le quadruple. Pour remédier i celle situation, le moyen qui paraît le plus simple consiste en la création de barrages de rétenue, permettant d'emmagasiner les eaux surabondantes dés crues pour les lâcher ensuite progressivement pendant les périodes de basses caux. Malheureusement, les barrages coutent fort cher, et il n'eśl d'ailleurs que rarement possible de trouver dans le bassin d'une rivière des emplacements favorables pour leur édification. Jusqu'à présent; là régularisation du régime des rivieres au point de vue industriel n'a pu se faire que dans des càs très rares, et notamment lơrsque l'on pouviait utiliser des lacs. L'exemple le plus remarquable est celuì du Rhốne dont le régime est régularisé à la sortie du Léman. Grâce à l'éhorme superficie du lac, il a été possible, en faisant rarier son niveau d'environ un mètre, de tripler le débit d'étiage di fléve en aval.

(1) La Durance, par Ivan WIrHELM: a la librairie Jules Rey 28, Grande-Rub, Grenoble (1913). Pride :30 ft.
Dènombreux barrages plus ou moins importants ont élé construits, dans les diverses parties du monde, mais principalement dans les pays chauds, pour fournir de l'eau a l'agriculture. On peut citer, dans cet ordre d'idées, les barrages qui ont été ćtablis en Espagne, en Algérie, dans l'Inde anglaise, en Egypte, el enfin aux Elats-Unis, dans la région aride, où le gouvernement américain fait construire depuis quelques années de gigantesques réservoirs, an moyen de barrages atleignant jusqu'à roo mètres de hauteur.

En France il cxiste, notamment dans la région du Contre, des barrages assez jmportants qui sond ulilisés principalement pour l'alimentation des villes el de divers canaux de navigation. ¿Les lecteurs de La Houille Blanche retrouveront dans les numéros de rgo6 à rgo8 les études de M. Betzer sur ces ouvrages). C'est soulement vers l'année $r 896$ que l'on a songé à la créalion de barrages dans l'intérêt do l'Agriculture, et c'est en Provence que la question est venue à se poser à la suite dé l'insuffisance maintes fois conslatée du débit de la Durance pour alimenter lous les canaux d'irrigalion qui sont dérivés de celle rivière.

A l'origine, on s'était préoccupé exclusivement des besoins de l'agriculture et c'est seulement plus tard que l'on a été amené à étudicr l'amélioration du régime de la Durance au point de vue industriel.

M. Wirmelm a ćlé appelé, en raison de ses fonclions, à ćludier la plupart des projets de barrage qui ont vu le jour dans le bassin de la Durance. Il a pu ainsi se rendre comple des besoins très divers, et parfois en opposilion d'inlérêts, qui cxistent dains colle région, au point de vue de l'alimentation des canaux d’irrigation, à celui du régime des usines el enfin a celui des distributions d'cau potable. Dans le but principal de faciliter la conciliation de tous los intérêts en présence, il a jugé utile de faire un exposé général de toules ces questions dans l'ouvrage dont nous venons de parler et dont noìs allons résumei ci-après les parties essenticlles.

\section{UTHLISATIONS INDUSTRIELLES ET AGRICOLES}

L'ouvrage, avons-nous déjà dit, débute par une monographie du bassin de Ja Durance, donnant da description des principales vallées et uné étude du régime de la rivière, avec des indications sur le climat et sur la géologie du bassin.

En raison du climat très sec de la Provence, Jes irrigations ont atteint, dans le bassin de la Durance, un développement, plus grand que dans n'importe quelle autre partie de la France. Dans chạcun des départements des Hautes el BassesAlpes l'irrigation absorbe une dizaine de mères cubes par seconde. Dans des Bouches-du-Rhône et Vaucluse le volume d'eau dérivé atteint go mètres cubes. Au total, c'est donc l'énorme débit de r ro mètres cubes par seconde qui est prélové sur le débit de la Durance et de ses aflluents pour les besoins des irrigations de la région. On pourrait même uliliser un débit plus important, car il cxiste encore de vastes lerrains incultes, principalement dans la Crau, que l'irrigation pernuetrait de mettro en valeur.

I'industrie de la houille blanchè a fait là son apparition beaucoup plus tard que dans les régions voisines de l'Isère cl de la Savoie, mais olle est appelée à prendre un très grand développement dans le bassin de la Durance qui offré à l'industrie des forces motrices abondantes. Dès à présent, il existe un certain nombre de grandes usines hydroclectriques, dont les plus puissantes sont colles de l'Argentière (35 ooo HP), de Ventavon (25 ooo HP) et de La Brillanne (15 ooo HP). L'auteur évalue à 600 ooo HP la puissance totale des usines existantes ou projetées dans le bassin de la Durance, chiffre qui pourrait vraisemblablement etre porté 
ì 850 ooo HP par la mise en cuvre de toutes les chules utilisables industriellement (puissance aux débils moyens).

Les utilisations agricoles et industrielles qui viennent d'être résumées succinclement justifient amplement la nécessité de faire des sacrifices pour améliorer le régime si variable de li Durance. Mais il y a encore une autre raison à invocuer en faveur de la création des barrages régulateurs. On sait que le lilloral de la Méditerranée manque d'eau el surtout d'eau potable. La ville de Marseille possède, il est vrai, une alimentation très abondante, mais en eau non potable, car celle qui est distribuée n'est autre que l'eau de la Durance, amenée par un canal à ciel ouvert de go kms de longueur, après une. simple décantation uars les bassins de SaintChristophe et de Réallort. Mais la plupart des autres villes, Touion, par exemple, manquent d'eau; il en est ainsi dans la plupart des communes rurales. Or il existe; à environ i20 $\mathrm{kms}$ de Marscille, -une source magnilique, 'dite "de Fonlaine-l'Evêque, qui débitc, cn basscs caux, 3 ooo litres par seconde, c'esta-dire plus qu'il n'en farit pour alimenter une population d'un million d'habitants. Le projet de dérivation de celte source cst déjà à l'étude, depuis longtemps, mais comme la source de Fontaine-l'Erêque fournit un appoint très important au Verdon, affluent de la Durance, on nó pourrait la dériver quaprès avoir offert une juste compensation aux intéressés du Verdon inféricur el de la basse Durance,ce qui ne pourra sefaire que par la crealion debarrages.

I.csintérêts en présence sonl, comme on le voit, très mul. liples, ef leur conciliation paraît nécessaire, non seulement pour éviter l'opposition de certains groupes d'intéressés, mais aussi pour parvenir a des solutions qui ne soicnt pas trop onéreuses. Il est certain que, d'une manic̀re gćnćrale, si un barrage ne doit servir qu'ì des besoins agricoles, sa dépense sera presque toujours hors de proportion avec les intérêts en jeu. Les pénuries d'eau de Ja basse Durance ne se produisent pas régulièrement chaque année. C'est à peine si le débit est insuffisant, pendant quelque temps, une année sur deux, et c'est seulement tous les s ou to ans quo les pénuries d'eau attcignent des proportions désastreuses. Dans ces condjtions, construire un barrage exclusivement pour des besoins agricoles, cela revient à faire payer très cher une sorte d'assurance contrc les reffels de la sécheresse. II n'en est pas tout à fait ainsi pour l'industrie, car il sc produit chaque année des débils d'étiage notablementinférieurs aux débits que les usines peuvent utiliser dans la plupart des cas.

Au point de vue industricl, les barrages offent donc une utilité régulière et permanente. Le mieux serait alors d'adopter des solutions permettant de concilier les intérêts de l'agriculture et ceux de l'industrie. C'est ce que M. Wilhelm s'est efforcé de faire dans l'étude du plan d'ensemble qu'il a ćtabli pour l'aménagement des reaux du bassin de la Durance. Le résultat cherché sera: obtenu par l'application du principe suivant : aménager chaque cours d'eau de manière à maintenir son débil aussi près que pos: sible du débit moyen. 'En procé dant ainsi, on donnera satisfaction aux besoins de l'industrie, car sub un cours d'eau dont le régime sem nolablement amélioré "par un ou plusieurs résenvoirs, il 'n'est pas excessif d'admettre que le débil maximum à utiliser dans los usi nes pourra atteindre le débit moyen du cours d'ean. L'agriculture pourra également recevoir salisfaclion si Ja régularisation du ré gime de la rivière principale' et de ses aflluènts est réaliséc sur ane ćchelle suffisante.

La justesse de ce principe est mise en relief; pour le bassin de la Durance, par l'examen des projets qui ont éte envisat

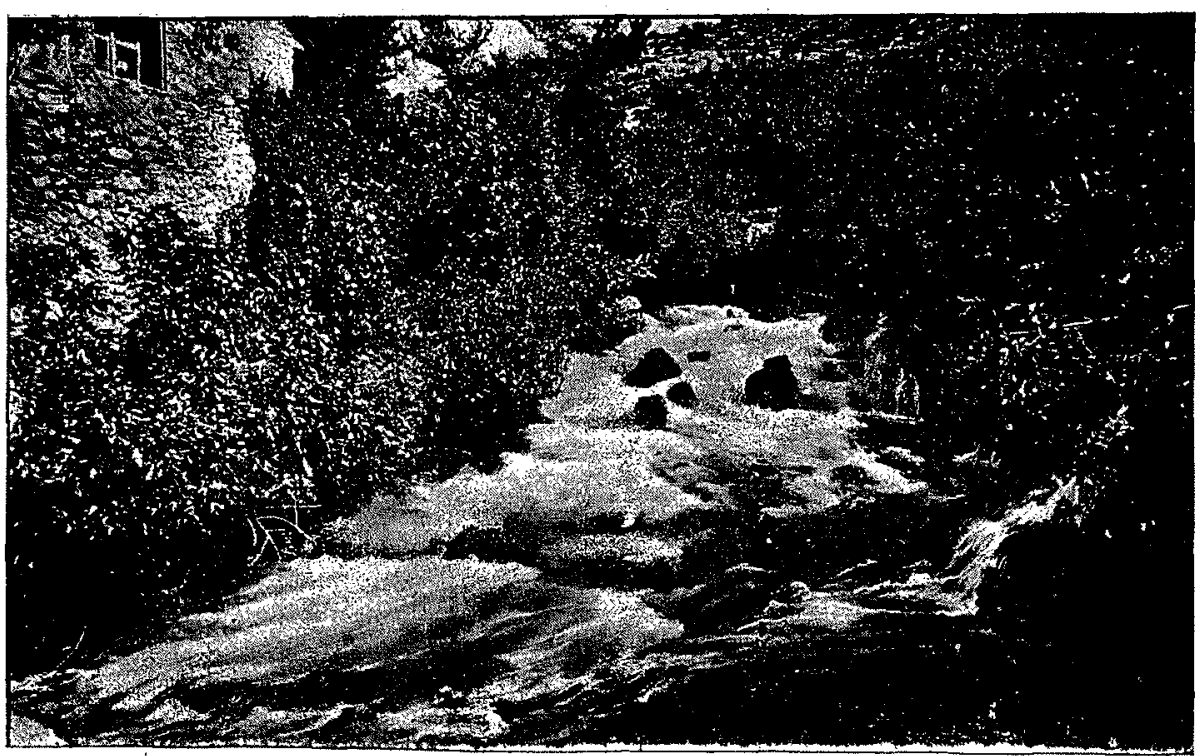

YUE dE LA SOURCE DE FONTAINE-L'EYÊUE gés. A la suite des coludes qui ont été réculées par l'Etat, l'Administration a été amcnée à préparer un projet de loi pour la créalion, sur le Verdon, d'un barrage assez important près dè Gréoulx. Le projet ne prévoyait que la satisfaction des intérêts agricoles et cela dan's des conditions telles; que loin d'être utile à l'industrie, ce barrage lui serait plutôt nuisible, ainsi qu'on va le voir. Le barrage en question retien drait $9^{5}$ millions do mètres cubes et co volume d'eau serait lâché pendant l'été; suivant les besoins. Dans une année très sèche, les lâchures pourraicnt atteindre 40 ì $45 \mathrm{~m}^{3}$, alors que l'on ne pourrait ntiliser iridustrigllement qu'un débit de ab $\mathrm{m}^{3}$ snviron, $\mathrm{b}$ 'aty. 
tre part, il était prévu que le remplissage du réservoir pourrail so faire, en partic, par un prélèvemenl sur les basses eaux de lhiver. Ainsi, au point de vue industricl, l'étiage sc trouverail aggravé, ct une partie seulement du volume d'eau accumulce pourrait êlre utilisée par les usines. Si, au contraire, lo,barrage de Gréoulx élait associé avè d'aulres barrages d'me importance suffisante, à créer dans diverses partics du bassin de la Durance, on aurait Ja possibilité de ne pas laisser couler pendant l'été, en aval de chacun d'eux, un dóbil supéricur au débit moyen du cours d'eau intéressé, tout en réalisant dans la basse Durance une augmentation du débit d'éliage suffisante pour donnér salisfaction ì lous les bosoins des canaux d'irrigalion. Avec un régime ainsi établi il scrait possible de faire concourir l'industric, dans une large mesure, aux frais d'établissement des barrages et d'alléger ainsi les charges de l'agriculture. rer obtenir en ce qui concerne le régime des cours d'eau. M. Wilhelm considèrc le reboisement comme un excollent moyen pour assurer la protection du sol contre l'érosion, ralentir l'écoulement dés caux et par' suite diminuer la torrenlialité et le charriage de matériaux qui en est la conséquence. Mais il ne croit pas que l'on puisse espérer obtenir une amélioration. de quelque importance du régime des cours d'eau. Il montre lout d'abord que le programme d'ensomble des roboisements, en parlie déjà réalisé, s'élendra au maximum à r/o ooo hectares, c'est-ì-dire à moins du $1 / 8^{\circ}$ de la surface du bassin de la Duranee. Il en conchu, qu'en supposant qu'on puisse mener à bien l'exéculion de ce vaste programme; le régine de la rivière ne pourra en être que faibloment modifié: D'autre part, la prédominance dans lo bessin de la Durance de terrains à peu près imperméables. lels que les marnes et les schistes, ne permet pas d'espérer

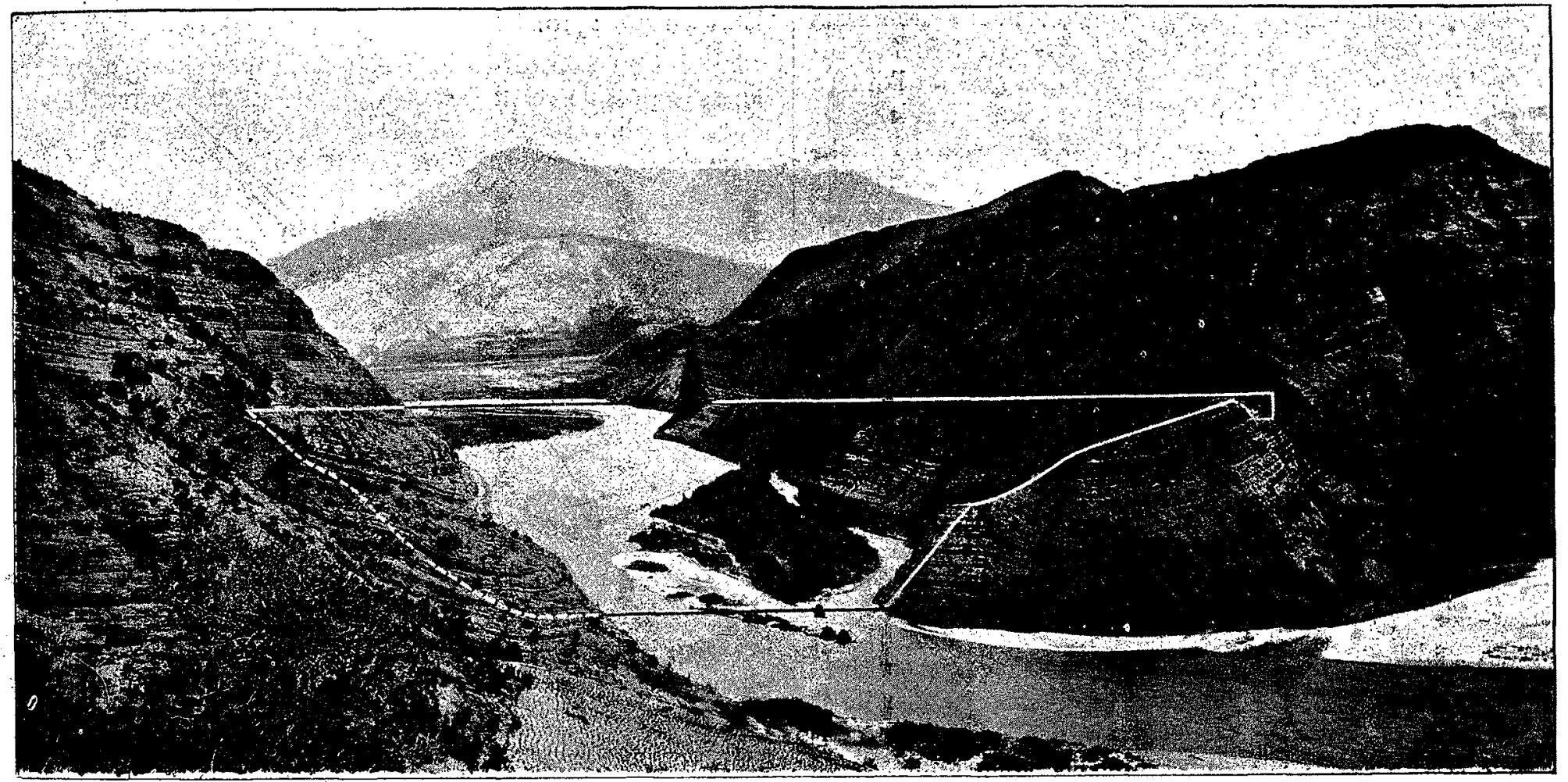

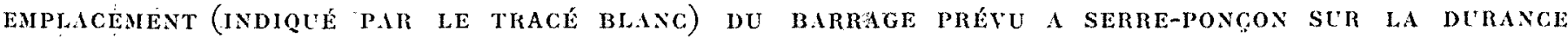

\section{CONDITUOS J'HTABLISSEMENT DES GRANDS BARBAFES}

Avart d'exposer le programme d'ensemble dont il préconise l'exécution, l'auteur a jugé nécessairc de fairc une étude de limportante question du charriage des matériaux solides par la Durance el ses aflluents. Il y a là, en effet, un sujet de graves préoccupations, on ce qui concerne l'avenir des barrages, car on sait que dans cetle région, en grande partic dimudía les torrents transportent beaucoup de limon et de graviers et que l'on pourrait donc craindre- Io comblement à bref délai des réservoirs créés à grands frais, si l'on ne prenail des mesures pour assurer l'évacuation des dépôts. ll est manin que la torrentialité de la pluparl des cours d'eau, qui st un vérilabie lléau de cetterégion, est de naturo à inspirer de sérieuses préoccupalions. L'auteur a cherché à connaître iussi exactement que possible l'étendue du mal, c'est-à-dire it déteminer les quantités de limon et de graviers pouvant the transportérs dans chaque cas. Pour romédier à ce mal, nn à próconisé le reboiscment à outrance des montagnes des Ilautes ot Bassosadlpes. Mi Wilhelm a étudié cette question

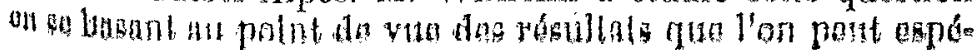

que la forêt puisse apporter une amélioration bien efficace an régime des sources. Si l'on veut améliorer le rógime de la Durance pendant les périodes de sécheresse, il sera donc indispensable de construire des barrages de retenuc et de faire los sacrifices nécessaires pour en assurcr le dévasement.

Lorsque l'on reut régulariser au moyen de barrages-réservoirs le régime d'un cours'd'eau, on rencontre généralement des difficultés assez grandes pour trouver des emplacements propices à l'établissement de ces ouvrages. Il faut toul d'abord que le rocher de fondation d'un grand barrage soil de bonne qualité, c'est-à-dire résistant, compact et étanche, el que sa profondeur sous les alluvions de la riviore ne soit pas excessive. Il faul aussi que la vallée s'ipanouisse suffisamment en amont pour permeltre l'accumulation d'un cube d'eau important, if rue cetle vallés puisse former une cuvette chanche. Enfin, il est nécessaire que les terrains compris dans le champ d'inondation puissent être acquis à un prix râisonnable, c'est-à-dire que la vallée soit peu habitéc ot que les bons terrains de culture y soient relativement

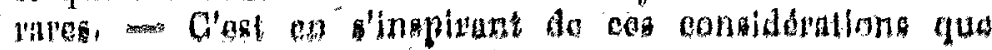


M. Wilhelm a proposé un programme d'ensemble comprenant les barrages qui lui ont paru les plus intéressants.

\section{Les grands barrages du bassin de la Durance}

Sur la Dưrance elle-même, il n'a été prévu qu'un seul barragé; mais quii serait d'importance exceptionnelle, celui de Serre-Ponçon, à 2 kmis en aval du confluent de l'Ubaye. Cet emplacement a été signalé vers 1860 , ì une époque où il était question de créer dans le bassin du Rhòne des réservoirs dont le seul but devait être d'empècher les inondations en emrragasinant les crues.

Sur les affluents do la Durance, on ne trouve, exception faite toutefois pour te Verdon, que peu d'emplacements favorables. M. Wilhelm en a signaló un dans les gorges du Guil, ì La Chapelue, ct deux dans la partic supérieure de la valléc de l'Ubaye, le premier au pont du Castelet et le second au lac du Paroird.

Le Verdon, qui a creusé des gorges étrnites dans des barres de calcaire jurassique, offre des emplacements très favorablis, car il arrive dans plusieurs cas qu'en amont de l'entrée d'une des ces gorges, la vallée s'épanouit considérablement.

Un premièr barrage qui a relenu particulièrement l'attention des Pouvoirs publics et des intéressís, pourrait être ćlabli, ainsi que nous l'avons déjà expliqué, à quelques kilomètres en amont de Gréoulx. Deux aütres ériplacements ont étế signalés par M. Wilhelm, l'un en aval de Castellane, à Caréjuan, l'autre en amont de la méme ville, à Cástillon.

Do plus, un ingénieur quii s'est spécialemient occupé de la question de la dérivation de Fontaine-l'Evêque a signalé drux emplacenents très remarquables, sur l'Artuby, affluent du Verdon, l'un à Comps et l'autre à La Martre.

Enfin, il convient de citer l'aménagement en résérvoir dú lac d'Allos; situé à 2200 mètres d'altitude dans le bassin supérieur du Verdon, qui avait été préconisé il y a déjà plus de 20 ans par M. PénIER, ingénieur en chef du Var, également dans le but d'offrir des compensations devant permettre la dérivation de Fontaine-l'Evếque.

Dans son programme d'ensemble, M. Wilhelm a compris tout d'abord les réscrvoirs de Serre-Ponçon, de Caréjuan, et de Gréoulx qui, établis sur la Durance elle-même ou sur le Verdon, auraient une action régulatrice énergique sur le régime de ces deux rivières. Il a cru devoir y ajouter les réscrvoirs de La Martre el d'Allos qui, bien qu'assez faiblèment alimentés, fourniraient un appoint précieux dans les années exceptionnellement sèches, tout en ne nócessitant que des frais insignifiants ou même nuls pour leur dévasement. Et ì la suite des discussions qui ont cu lieu contre parlisans et adversaires de l'améagement du Guil, et qui ont finalement occasionné le vote par la Chambre des Députés d'une disposition ayant pour objet d'assurer la protection des sites constitućs par certains cours d'eau pittoresques (1), la création du barrage de La Chapelue apparaît également comme intéressante.

Leis caractéristiques des barrages compris dans le programme eri question sont résumées dans le tableau suivant :

\begin{tabular}{|c|c|c|}
\hline INDICATION DES BARERIGES & 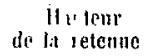 & 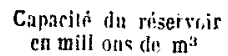 \\
\hline Barrage de Serre-Ponçon...... & $85 \mathrm{~m}$ & 607 \\
\hline Barrage de Caréjuan.......... & $70 \mathrm{~m}$ & 140 \\
\hline Barrage de Gréoulx......... & $54 \mathrm{~m}$. & 95 \\
\hline Barrage de La Martre........ & $50 \mathrm{~m}$. & 80 \\
\hline Aménagement du lac d'Allos... & $30 \mathrm{~m}$. & 40 \\
\hline Barrage de La Chapelue...... & I IO $\mathrm{m}$. & 30 \\
\hline
\end{tabular}

(1) Voir à re sujet l'arlicle de M. Paul Bouc.nular (II. B. mars 1913):

\section{Le grand barrage de Serre-Ponçon}

Ce barrage donnerait naissance à un lac artificiel qui occuperait une longueur de $\mathbf{r} 5 \mathrm{kms}$ dans la valléé de la Durance et de $6 \mathrm{kms}$ dans celle de l'Ubaye. Sa superficie serait de i 500 hectares. L'examen géologique auquel il a été procédé a démontré l'étanchéité de la cuvette. De plus, celle-ci est formée par des terrains de peu de valeur et les habitations y sont assez rares. Par contre, il y aurait d'assez importants travaux à faire pour le rétablissement des voies de communications comprises dans le champ d'inondation : reconstruire 35 kilomètres environ de routes nationales, et devier sur 6 kilomètres le chemin de fer de Gap à Briançon. De plus, il a été nécéssaire d'envisager la déviation du chemin de fer de Chorges a Barcelonnette, dont le tracé primitif se trouvait dans la cuvette du futur lac sur un parcours d'une dizaine de kilomitres. On retrouvera la description ct le plan de cel ouvrage dans La. Houille Planche de décembre rgı.

La plus grande difficulté qu'a rencontrée jusqu'à présent la rćalisation de ce projet provient de l'extrême profondeur du rocher de fondation sous le lit de la Durance, à l'emplacenient du barrage. Quelques sondages, avaient été exécuté̉s vers 1860 , et l'on avait cru pouvoir en conclure que l'épaisscur des alluvions ne dépassait en aucun point une dizaine de mètres. Mais lorsqu'en $i 8_{9} 6$ la question des barrages fut mise à l'étude en vue de remédier aux pénuries d'eau de la basse Durance, de nouveaux sondages furent exécutés par l'Etat avec le concours des départements intéressés. Ces soñdages, dont la direction fut confiée à M. Wilhelm, alors ingénieur à Gap, furent terminés en $I 89 g$. Le rocher fut reconnu à des profondeurs de ro à r2 mètres, maìs à des distances n'excédant pas une trentaine de mètres à partir des borges. Un dernier sondage, entrepris au milieu de la Durance, traversa $43 \mathrm{~m}$. d'alluvions. La sonde rencontra alors une forte résistance, mais, par suite de circonstancès aceidentelles, il ne fut pas possible d'éclaircir ce point d'une manière indiscutable. M. Wilhelm dressa néanimoins l'avantprojet d'un barrage de $50 \mathrm{~m}$. de hatuteur utile qui auratit permis de retenir 186 millions de mềtres cuibes d'eau. Măls l'Administration recula, à cette époque, devant les difficuiltés et les dépenses d'un tel projet et prescrivit de faire une étude d'ensomble pour déterminer les autres emplacements favorables pouvant exister dans le bassin de la Durance. Cette étude aboutit, comme nous l'avons indiqué, à la prise en considération du projet du barrage de Gréoulx.

Mais, en présence des difficultés que rencontrait l'aménagement du Verdon, au double point de vue de l'amélioration du régime de la basse Durance et de la dérivation des eaux de Fontainc-l'Evêque, M. Wilhelm eut l'idée de reprendre le projot du barrage de Serre-Poncon, en portant la hauteur de la retenue de 50 à $85 \mathrm{~m}$. Il a exposé son nouveau projet

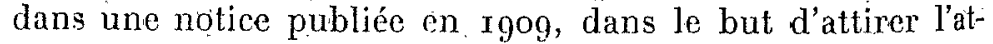
tenlion des intéressés et des pouvoirs publics sur les intérêts considérables qui lui paraissaient s'attacher à la réalisation dé cc projet. Il y avait une certaine urgence à prendre une décision à cet égard, car l'exécution prochaine du chemin de fer de Bancelonnette revait apporter un obstacle à peu prês insurmontable à lá réalisation du projet de Serre-Ponçon. Le Conșeil général des Hautes-Alpes demanda alors que des études fussent faites en vue de la déviation de ce chemin dé fer. Peu après; les divers groupements industriels intéressés à l'amélioralion du régime de la Durance constituaient une Société sous le nom de "Société pour la Régularisation de la Durance "; dont l'objet élait d'étudier; de concert divec 
YElat et sous son contròle, le projel du barrage de ScrePonçon, dont elle demandait alors la concession.

$\Lambda$ près une étude approfondie de la question, le Ministre des Travaux publics décida, cu rg r r, la dévialion du chemin de fer de Barcelonnelte. Le nouveau lracé franchira la valléc de la Durance à un niveau supérieur au maximum prévu pour celui du futur lac, au moyen d'un grand viaduc qui aura 7 oo $\mathrm{m}$. de Jongueur et $68 \mathrm{~m}$. de hauteur.

Il ful décidé égalemenl de procéder à de nouvenux sondages, dans te but d'obtenir des renseignements absolument certains sur la nature et la posilion du rocher de fondation. Ces sondages ont élé commencés au mois de septembre dernier et se poursuivent depuis, sans interruption. On a creusé tout d'abord, sur la rive droite de la Durance, un puits dans le

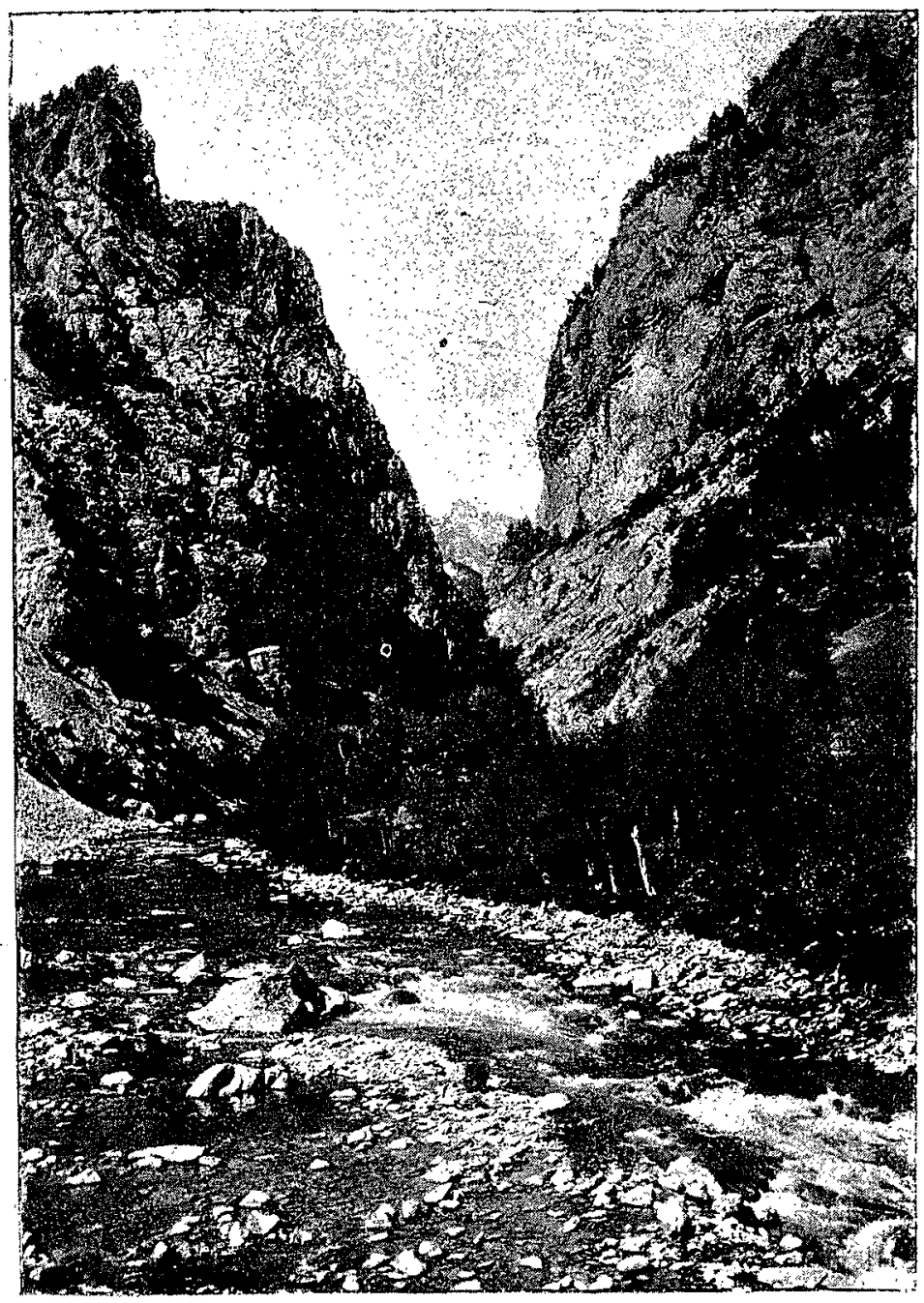

LES GORGLS DU GEll A LA CHAPELE

rocher que lon a descendu jusqu'it 57 m. on conlrebas du nivealu de l'éliage; du fond de ce puils on a fail partir ensuite une galerie à peu près horizonlale qui se prolongera sous le dit de la rivière jusqu'à une trentaine de mètres audelit de l'axe de celle-ci. Cetle galerie alleignait, le 29 mars dermier, une Jongucur de 72 mètres, constamment dans to rocher. Lorsque l'on aura avancé encore d'une trenlaine de mètres on aura obtenu la preuve, si celle galeric continue à se maintenir dans le rocher, que la profondeur maxima de celui-ci sera jnférieure à 55 mètres. C'est un point sur lequel on scra prochainement fixé et il y a lieu d'espéxer, si les résullats oblenus conlinuent à être favorables, que rien no sopposera à ce que l'on donne suile à cel imporlant projet. Son exécution présentera évidemment des difficultés considérables. Il est prévu que l'on déviera la Durance dans deux grands souterains at moyen dun puissant barrage de garde. La fouille sera ainsi exécutéc entièrement à ciel ouvert. En raison de la nature très peu perméable des alluvions inf́rieures du lit de la Durance, on comple que l'on pourra exécuter les épuisements sans difficultés excessives.

Rien n'a encore élé arrêté en ce qui concerne le mode de construction de l'ouvrage. Il a été question de prévoir un barrage en béton armé, mais il faut bien ieconnaitre que ce mode de construction pourrait, à l'heure actuelle, etre jugé trop audacicux pour un ouvrage d'une hauleur aussi exceptionnelle. Il semble plus indiqué de faire un barage massif entièrement fondé sur le rocher, en maçonnerie ordinaire, ovec revêtement en moellons d'appareil, ou, mieux 'ncore, tout simplement en béton de ciment. Le cube de la masonnerie atteindrait le chiffre formidable de un million de mètres cubes.

Le barrage de Serre-Ponçon, de $85 \mathrm{~m}$. de hauteur utile, permettrait d'aménager une force motrice très importante. Toutefois, comme il ne serait pas admissible de laisser tomber la chule à zéro, l'auleur du projet a prévu que l'ón sicrifiera les $40 \mathrm{~m}$. inférieurs de la retenue. On perdra ainsi uno centaine de millions de mètres cubes sur la capacité du réservoir, mais on aura une chule qui variera entre un minjmun de $10 \mathrm{~m}$. el un maximum de $85 \mathrm{~m}$. De plus, on pourra pendant longlemps loger dans la partie inféricure du réservoir les alluvions apportées par Ja Durance et par l'Ubaye.

Ja question du dévasement du réservoir est particulièrement importante. M. Wilhelm, qui a fail à ce sujyl des recherchos très étendues, a évalué à plus de deux millions de mètres cubes le volume annuel des limons, et à cnviron $200000 \mathrm{~m}^{3}$ colui des graviers qui entreront en moyennc chaque annéc dans le réservoir.

Arec les dispositions qui onl élé prévues, on disposera en nombre rond d'une capacité ulile de 500 millions de nètres cubes. M. Wilhelm a fait dans son ouvrage une élude délaillée de l'inlluence que pomrait avoir le réservoil de SorrePoncon sur le régime de la Durance en aval. Actucllemenl, le débit de la rivièe descend comanmment, en hiver, a $25 \mathrm{~m}^{3}$ el execpliomellement ì 20 ol même $19 \mathrm{~m}^{3}$. Je débit moyen de la Durance à Serre-Ponçon est approximalivement de $80 \mathrm{~m}^{3}$. C'ost ce chilfre que $\mathrm{M}$. Wilhelm a adoplé commo devant être de maximum du débit à laisser couler, en aval du barrage, pendant les périodes de basses raux. Co débil, qui me dépasse pas colui pouvanl che ulilisó avantagensement par l'industrie, permellra de donner salisfaction aux besoins de l'agrieullure. 1 cel effel, il esl prévu que, ohaque ammé, le débit de la Durance cn aval du barrage sera maintenu a au moins $80 \mathrm{~m}^{3}$ pendant les mois de juillet, août el sophonbre le débit de la Durance se trouvera ainsi augruenté considérablement pendant l'été, el dans les ammécs axceptionnellement sèches on aura une amélioration qui pourra alloindre jusqu'ì fo el même $45 \mathrm{~m}^{3}$. Pendant les basses caux d'hiver, l'amélioration du débil sera réaliséc d'après le volume d'eau disponible dans le réscrvoir et en teranl comple des prévisions que l'on pourra élablir pour le printemps suivant, d'après l'importance plus ou moins grande des réserves de neige qui existeront dans les montagnes.

M. Wilhelm produit des tableaux qui résument les éludes qu'il a failes pour la période des 20 années ćconlées de 189 ? à Ig I . Ces tableaux permettent de se rendre compte des amélioralions considérables qui scraient apportées an régime de la Durance. le débil d'étiage se mainliont labituclloment entre 5o et $60 \mathrm{~m}^{3}$ el descend exceplionnellement à fo m.3. De plus, le ccefficient d'utilisation des dérivations industrielles serait augmenté dans une forte proportion. 
La chute du barrage, ancénagée pour un débit maximum de $80 \mathrm{~m}^{3}$, atteindrait uno puissance maxima de $70000 \mathrm{HP}$ et une puissance moyenne de 50 ono IlP.

La régularisal ion de la Durance en aval permetla de récupérer une quantité d'énergie de plus de $50 \%$ millions de kilowatts-heure par an, lorsque toutes les chutes prévues, d'une hauteur netle tolale d'environ 400 mètres, auront été complètement amẻnagées.

La dépense a faire pour réaliser ce projel n'est pas encore exartement connue, mais, cu s'efforcant de compter Jargement, on a été amené à cnvisager une dépense totale de 65 millions, y compris le cout de l'usine du barrage. Celte dépense n'est pas hor's do proportion avec Jes résultats à obteniir, surtout si l'on tient compte de l'intérêt considérable qui s'altacho à la suppression des pénuries d'ean de la basse Durance el à l'exlension érentuclle des arrosages.

\section{Les barrages sur le Verdon}

Nous avons déjà donné danis La Houille Blanche la description a projet du barrage de Gréoulx (mai rgro). Le barrage de Caréjuan serail réalisé dans les inêmes conditions, c'est-ì-dire avec des disposilions analogues à celles prévues pour lo barrage de Serre-Ponçon. On sacrilierait également la partic inférieure de la retenue dans le but d'avoir une chute ulltisable industriellement. La capacité utile de co résenvoir serail ainsi rédnile a s 7 millions de mètres cubes.

Fn complélant les réserves de Carójuan par l'appoint, dtuns les années sìches, des réservoirs de la Martre et d'Altos, on chbliendrail nne régularisation très sérieise du régimne du Verdon, qui sorait encore acecrituéce en aval par le réservoir de Gréculx. Fin rue de préeiser relle régnlarisation, M. Whlhelm a fail des calculs qu'il a appliqués à la période de 38 amnées qui s'est écoulée de 1874 à xg I t, el qui liti ont pemnis d'arriver a ta conclusion suivante :

Alors que le débit du Verdon, ì l'emplacement du bar-

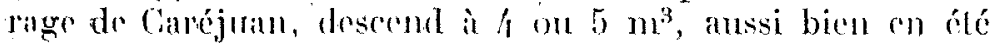
qu'ert hiver, on obtiondrai, all moyer des réservoim projetés, un débil mininum de ao $\mathrm{m}^{3}$, pendant les lons mois d'eté, el do $15 \mathrm{~m}^{3}$ pendant les antres mois. On réaliserait ainsi une régularisation très intéressante du Verdon, au double point de vue agricole el industricl. En admoltant que l'on réalise de projel de dérivation de la souree de Fon-

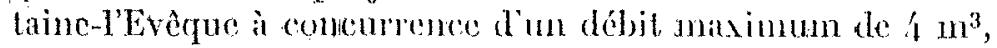
ref que l'ou veuille plus lart alfecter un débit supplémentaire de $3 \mathrm{~m}^{3}$ a des irrigalions ì réaliser dans le département du Vä, c'est-a-dire à faire sortir du bassin du Verdon un débit continu de $7 \mathrm{~m}^{3}$, on pourrait néammoins apporter cucore une certaine amélioration au débit d'étiage du Verdon vers som confluent arec la Durance, amélioralion qui s'éleverait jusqu'i 7 hu 8 m $^{3}$ dans les années exceptionnellement sèches.

Nous ne saurions terminer ce pàle aperçu du travail de M. Whaterm sans lui adresser l'hommage de nos meilleurs veux pour lo suecès de l'entreprise qu'il próconise. Elle est de celles qui contribucront à l'accroissement de nolve oulitlrrge national et ì ce titre mérite l'encouragement de tout homme qui fail généreuscucnt oflont pour meltre en auve nos richesses naturelles.

lin dr. précedentes éludes, nous avons montre de quel intérel doit che pour le développenent de la prospérité conomique de nolve pays, la réalisation des grands projets d'aménagement da Haut-Bhône d'abond et ensuile du Rhône se Lyon à la mer. La régutarisation de la . Durance est ane wure du même ordse de grandeur, devant êlre mestéc à bonne fin dans lo mème temps que les précédentes, parec qu'elle les complète.

Il faut des centaines de millions, diratton, pour exicuter ces grandioses entreprises st ce n'est point de sitôl que l'industrie sera capable d'absorber les centaines de mille chevaux qu'elles erécront. Assurément, ces objections méritent qu'on s'y arrête; mais on ne doil s'y arrêter que pour fairc l'exanen très complet des solutions techniques proposécs avant d'engager sur l'une d'elles les finances publiques, car pour ce qui est des avantages économiques devant résulter de l'exéculion de ecs grandes entreprises, la question n'est plus discutable. la France importe annuellement 20 millions de tomes de charbons, tandis que la houille blanche, dont nous avons de si puissanles réserves, devient chaque jour d'un enploi plus pratique dans les industries de l'éclairage, de la foree motrice, de la traction, de la chimie el de la métallurgic. Que J'on songe donc que le coût du charbon irat toujouss en s'élevant, pendant qu'au conleaire te prix de revient de la houille blanche iria en s'abaissant an fur tet à mesure de l'amortissenent des frais de premier établissement. Il importe d'assurer l'avenir !...

Qui donc modiliera chez nous cette mentalité de par larjuclle le capital argent est considéré conme un instrument de travail plus productif à l'étranger qu'en France? Les ports et les chemins de fer des Etats de l'Amérique du Sud, le développement de la civilisation et du commeree dans les pays d'Orienl, sont cerles des entreprises dignes d'intérêt, mais à un degré moindre, nul ne le contestera, que ces améliorations à notre outillage national. Quand done nos grands élablissenents de crédit en viendront-ils ì celte formule : L'or de France à l'outillage français!

E.-F. CÜ'TE.

\section{HYDRAULIQUE}

\section{Gonstruction rationnelle des tuyaux d'aspiration en Béton}

Depuis quelques années, les ingénieurs hydrauliciens se sont allachés à récupérer une parlie de l'énergie perdue dans l'évacuation de l'eau des turbines par une construetion plus rationnelle des conduits de sortie. L'emploi du bóton a permis de donner facilement a ces conduits des formes diminuant les pertes de charge cn cet endroit.

l.importance de celle récupération est d'autant plus grande que la chute à utiliser est plus faible; le pourcentage perdu par la vitesse résiduclle de l'eau étant relativement plus élevé pour les basses chules.

Nous attons cxposer sommairement le procédé inventé par l'ingénieur R. Dubs, de Zürich.

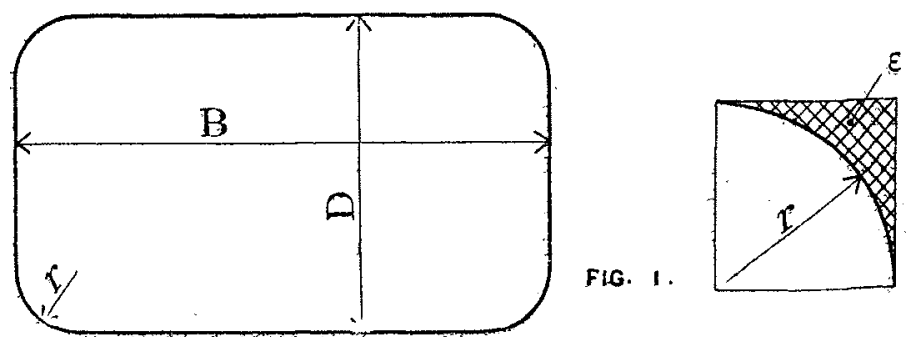

Appelons $D$ la laxgcur d'une section du luyau considét; $B$ sa longueur, $r$ le rayon des antrondis; 1 la section atilisable $f$ a pour valeur:

$$
f=B D-4
$$

